\title{
THE EQUIVALENCE OF HILBERT AND MUMFORD STABILITY FOR VECTOR BUNDLES*
}

\author{
ALEXANDER SCHMITT'
}

\begin{abstract}
We prove the equivalence of the notions of Hilbert (semi)stability and Mumford (semi)stability for vector bundles on smooth curves for arbitrary rank.
\end{abstract}

Introduction. Let $d, g$, and $r$ be fixed positive integers, $W$ a complex vector space of dimension $p:=d+r(1-g)$, and $\mathfrak{G}=G(W, r)$ the Grassmannian of $r$ dimensional quotients of $W$. On $\mathfrak{G}$, there is the universal quotient

$$
W \otimes \mathcal{O}_{\mathfrak{G}} \longrightarrow E_{\mathfrak{G}}
$$

which induces a surjection $\bigwedge^{r} W \otimes \mathcal{O}_{\mathfrak{G}} \longrightarrow \bigwedge^{r} E_{\mathfrak{G}}$, defining the Pluecker embedding $\mathfrak{G} \hookrightarrow \mathbb{P}\left(\bigwedge^{r} W\right)$. Now, let $C \hookrightarrow \mathfrak{G}$ be a smooth curve of genus $g$ such that $E_{C}:=E_{\mathfrak{B} \mid C}$ has degree $d$. Then, $C$ gets embedded into $\mathbb{P}\left(\bigwedge^{r} W\right)$ as a curve with Hilbert polynomial $P(m)=\chi\left(\left(\bigwedge^{r} E_{C}\right)^{\otimes m}\right)=d m+(1-g)$. From the restriction $W \otimes \mathcal{O}_{C} \longrightarrow E_{C}$ of the universal quotient to $C$ we derive homomorphisms $\wedge^{r} W \otimes \mathcal{O}_{C} \longrightarrow \bigwedge^{r} E_{C}$, and for all $m \geq 1$

$$
\psi_{C}^{m}: S^{m} \bigwedge^{r} W \longrightarrow H^{0}\left(\left(\bigwedge^{r} E_{C}\right)^{\otimes m}\right)
$$

If $\psi_{C}^{m}$ is surjective (as will be the case for large $m$ ) and $h^{0}\left(\left(\bigwedge^{r} E_{C}\right)^{\otimes m}\right)=P(m)$, this yields

$$
\varphi_{C}^{m}:=\bigwedge^{P(m)} \psi_{C}^{m}: \bigwedge^{P(m)}\left(S^{m} \bigwedge^{r} W\right) \rightarrow \mathbb{C}
$$

We call $C$ or, abusively, $E_{C} m$-Hilbert (semi/poly)stable, if $\psi_{C}^{m}$ is surjective, $h^{0}\left(\left(\bigwedge^{r} E_{C}\right)^{\otimes m}\right)=P(m)$, and the point $\left[\varphi_{C}^{m}\right]$ in $\mathbb{P}\left(\bigwedge^{P(m)}\left(S^{m} \bigwedge^{r} W\right)\right)$ is (semi/poly)stable w.r.t. the natural action of $\mathrm{SL}(W)$ on that space, and Hilbert (semi/poly)stable, if it is $m$-Hilbert (semi/poly)stable for all $m$ sufficiently large. This is now a new stability concept for the vector bundle $E_{C}$ entering in competition to classical Mumford stability. It goes back to Gieseker and Morrison ([4], [5]). Its main motivation is to obtain an alternative compactification, called Hilbert stable compactification by Teixidor [11], of the universal moduli space of semistable vector bundles of rank $r$ and degree $d$ over $\mathfrak{M}_{g}$, the moduli space of smooth curves of genus $g$, by letting $C$ vary and degenerate in $\mathfrak{G}$. In contrast to the slope stable compactification of Pandharipande [9] which involves torsion free sheaves on singular curves, this compactification would take place entirely in the realm of vector bundles. Its potential usefulness is illustrated by the paper [5] where Hilbert stable vector bundles on a nodal curve are used to prove a conjecture of Newstead and Ramanan on the moduli space of stable rank two bundles over a smooth curve. In order to make such a theory

\footnotetext{
*Received August 4, 1999; accepted for publication August 31, 1999.

†Universität GH Essen, FB6 Mathematik \& Informatik, D-45117 Essen, Deutschland (alexander.schmitt@uni-essen.de). Supported by a grant of the Emmy Noether Institute at Bar-Ilan University.
} 
work, the objects one starts with, namely Hilbert and Mumford stable vector bundles on smooth curves, have to be same. Thus, one must show (1) that every stable vector bundle of rank $r$ over a smooth curve $C$ of sufficiently high degree $d$ gives rise to an embedding of $C$ into $\mathfrak{G}$ and (2) that for $C \hookrightarrow \mathfrak{G}$ Hilbert and Mumford stability for the bundle $E_{C}$ coincide. The first point follows from a recent theorem of Butler [2] (see 1.1.1 below), and (2) has been established in the rank two case by Gieseker and Morrison [4]. It is the aim of the present note to settle the general case, i.e., prove

THEOREM. Fix $g$ and $r$, then there is a constant $d_{0}$ such that for every $d \geq d_{0}$ and every complex vector space $W$ of dimension $p=d+r(1-g)$ there exists a constant $m_{0}=m_{0}(d, g, r)$ such that for all $m \geq m_{0}$ the following holds true: Let $C \hookrightarrow G(W, r)$ be a smooth curve of genus $g$ and $W \otimes \mathcal{O}_{C} \longrightarrow E_{C}$ the restriction of the universal quotient to $C$. Assume $W \longrightarrow H^{0}\left(E_{C}\right)$ is an isomorphism and $\operatorname{deg}\left(E_{C}\right)=d$. Then $C$ is $m$-Hilbert (semi/poly)stable, if and only if $E_{C}$ is a (semi/poly)stable vector bundle.

Note that both the condition of Mumford and Hilbert stability can be formulated as stability requirements on the quotient $W \otimes \mathcal{O}_{C} \longrightarrow E_{C}$. Therefore, it is a natural idea to look at the SL $(W)$-action on (some open part of) the quot scheme of quotients of $W \otimes \mathcal{O}_{C}$. As it turns out both stability conditions give rise to the same linearized line bundle on this open part of the quot scheme. If the parameter space were projective, this would settle the problem. Since this is not the case, we have to see how the curve $C$ with $E_{C}$ semistable and Hilbert semistable might degenerate in the set of Hilbert semistable points. In turns out that the degeneration is roughly $C$ with some rational components attached, a case which can be excluded by an adaptation of an argument from [11]. In other words, the locus of smooth curves $C^{\prime}$ which are isomorphic to $C$ such that $E_{C^{\prime}}$ is semistable is closed in the locus of Hilbert semistable points. This is now as good as the projectivity of the parameter space and one can conclude by standard methods in Geometric Invariant Theory. Our proof therefore avoids completely any non-trivial computation.

\section{Preliminaries.}

1.1. Review of some aspects of the theory of semistable vector bundles. A vector bundle over a smooth curve $C$ is called (semi)stable, if it satisfies $\mu(F)(\leq) \mu(E)$ for all non-trivial proper subbundles $F \subset E$, and polystable, if $E$ is isomorphic to a direct sum of stable bundles all of which have the same slope.

The following is a recent generalization to semistable vector bundles of a result of Mumford on line bundles.

THEOREM 1.1.1 (Butler [2]). Let $E$ and $E^{\prime}$ be semistable vector bundles on the smooth curve $C$ of genus $g$. Assume $\mu(E)>2 g$ and $\mu\left(E^{\prime}\right) \geq 2 g$. Then the homomorphism

$$
H^{0}(E) \otimes H^{0}\left(E^{\prime}\right) \quad \longrightarrow \quad H^{0}\left(E \otimes E^{\prime}\right)
$$

is surjective.

From this, one infers (see [12])

CoROLlARY 1.1.2. Let $E$ be a semistable vector bundle of rank $r$ on the smooth curve $C$ of genus $g$ with $\mu(E)>2 g$. Then, the homomorphism

$$
\bigwedge^{r} H^{0}(E) \quad \longrightarrow \quad H^{0}\left(\bigwedge^{r} E\right)
$$


is surjective.

Note that under the assumptions of 1.1.2, $H^{1}(E)=0$. So, Corollary 1.1.2 shows that the quotient $H^{0}(E) \otimes \mathcal{O}_{C} \longrightarrow E$ defines an embedding of $C$ into $G(W, r)$ where $W$ is a complex vector space of dimension $\operatorname{deg}(E)+r(1-g)$.

Proposition 1.1.3. Fix $g$ and $r$. Then there is a constant $d_{1}>2 g$, such that for every curve $C$ of genus $g$ and every vector bundle $E$ of rank $r$ and degree $d \geq d_{1}$ the following conditions are equivalent

1. E is a (semi)stable vector bundle.

2. $h^{0}(F) / \mathrm{rk} F(\leq) \chi(E) / r$ for all non-trivial proper subbundles $F$ of $E$.

3. $\chi(E) / r(\leq) h^{0}(Q) / \operatorname{rk} Q$ for all non-trivial proper quotient bundles $Q$ of $E$.

Proof. This is standard. See [8] or [7]. From the proof one can easily determine an explicit value for $d_{1}$.

1.2. Properties of semistable points. Let $X$ be a quasi projective scheme on which the reductive group $G$ acts. Suppose this action comes with a linearization in an ample line bundle $A$. Then, the open sets $X^{s s}$ and $X^{s}$ of semistable and stable points are defined. Furthermore, a semistable point $x$ is called polystable, if its orbit is closed in $X^{s s}$. The set of polystable points will be denoted by $X^{p s}$. Now, assume that $X$ is projective. For any point $x \in X$ and any one parameter subgroup $\lambda$ of $G$, we define $\mu_{A}(x, \lambda)$ as minus the weight of the $\mathbb{C}^{*}$-action induced by $\lambda$ on the fibre of $A$ over the point $\lim _{x \rightarrow 0} \lambda(z) \cdot x$. The Hilbert-Mumford criterion then says that a point $x$ is (semi)stable if and only if $\mu_{A}(x, \lambda)(\geq) 0$ holds for every one parameter subgroup $\lambda$ of $G$. Moreover, $x$ is polystable if and only if it is semistable and a fix point for every $\mathbb{C}^{*}$-action coming from a one parameter subgroup $\lambda$ with $\mu_{A}(x, \lambda)=0$.

Next, suppose we are given two representations $\rho_{1}: G \longrightarrow \mathrm{GL}\left(V_{1}\right)$ and $\rho_{2}: G \longrightarrow$ $\mathrm{GL}\left(V_{2}\right)$ of the reductive group $G$ on the finite dimensional $\mathbb{C}$-vector spaces $V_{1}$ and $V_{2}$. This yields an action of $G$ on $\mathbb{P}\left(V_{1}\right) \times \mathbb{P}\left(V_{2}\right)$ together with natural linearizations in $\mathcal{O}\left(t_{1}, t_{2}\right)$ for all $t_{1}, t_{2}>0$. The corresponding set of (semi/poly)stable points depends only on the parameter $\vartheta:=t_{1} / t_{2} \in(0, \infty)$ and will be denoted by $Q_{\vartheta}^{(s / p) s}$. We also define $Q_{0}^{(s / p) s}$ and $Q_{\infty}^{(s) s}$ as the preimage of the (semi/poly)stable points under the projection onto $\mathbb{P}\left(V_{1}\right)$ and $\mathbb{P}\left(V_{2}\right)$, respectively. Then, the following properties are well known and easy to see ([13], [10]): There exists a finite number of critical values $\vartheta_{1}, \ldots, \vartheta_{s} \in(0, \infty)$ such that, settting $\vartheta_{0}=0$ and $\vartheta_{s+1}=\infty$, for $i=1, \ldots, s+1$ and given $\vartheta, \vartheta^{\prime}$ in $\left(\vartheta_{i-1}, \vartheta_{i}\right)$

$$
\begin{aligned}
& Q_{\vartheta}^{(s / p) s}=Q_{\vartheta^{\prime}}^{(s / p) s} \\
& Q_{\vartheta}^{s s} \subset Q_{\vartheta_{i-1, i}}^{s s} \\
& Q_{\vartheta}^{s} \supset Q_{\vartheta_{i-1, i}}^{s}
\end{aligned}
$$

Now, let $X$ be a $G$-invariant closed subscheme of $\mathbb{P}\left(V_{1}\right) \times \mathbb{P}\left(V_{2}\right)$, and set $X_{\vartheta}^{(s / p) s}:=$ $Q_{\vartheta}^{(s / p) s} \cap X, \vartheta \in[0, \infty]$.

LEMMA 1.2.1. Suppose that there is an $n>0$, such that for every point $x \in X_{0}^{s s}$ and every one parameter subgroup $\lambda$ of $G$

$$
n \cdot \mu_{\mathcal{O}_{\left(V_{1}\right)}(1)}\left(\lambda, \pi_{1}(x)\right) \geq \mu_{\mathcal{O}^{\left(V_{2}\right)}(1)}\left(\lambda, \pi_{2}(x)\right) .
$$


Then, for $\vartheta \in\left(0, \vartheta_{1}\right)$, also

$$
X_{0}^{(s) s}=X_{\vartheta}^{(s) s}
$$

Proof. The stated condition clearly implies $X_{\vartheta}^{s} \subset X_{0}^{s}$, and thus, by (1.3), $X_{\vartheta}^{s}=$ $X_{0}^{s}$.

There is a surjective morphism $X_{\vartheta}^{s s} / / G \stackrel{\varphi}{\longrightarrow} X_{0}^{s s} / / G$ which is by our assumption an isomorphism over $X_{0}^{s}=X_{\vartheta}^{s}$. Thus, $X_{\vartheta}^{s s} / / G \backslash X_{\vartheta}^{s} / / G$ maps onto $X_{0}^{s s} / / G \backslash X_{0}^{s} / / G$ which means that for every point $x \in X_{0}^{s s} \backslash X_{0}^{s}$ there exists a point $x^{\prime} \in X_{\vartheta}^{s s} \backslash X_{\vartheta}^{s}$ with $\varphi\left(\left[x^{\prime}\right]\right)=[x]$. Choose $x^{\prime} \in X_{\vartheta}^{p s}$. We claim that $x^{\prime}$ also lies in $X_{0}^{p s}$. Indeed, let $\lambda$ be a one parameter subgroup with $\mu_{\mathcal{O}_{\mathbb{P}\left(V_{1}\right)}(1)}\left(\lambda, \pi_{1}\left(x^{\prime}\right)\right)=0$. By the assumption and the fact that $x^{\prime} \in X_{\vartheta}^{s s}$, we must also have $\mu_{\mathcal{O}_{\mathbb{P}\left(V_{2}\right)}(1)}\left(\lambda, \pi_{2}\left(x^{\prime}\right)\right)=0$, and hence $\mu_{\mathcal{O}\left(t_{1}, t_{2}\right)}\left(\lambda, x^{\prime}\right)=0$ for all $t_{1} / t_{2} \in\left(0, \vartheta_{1}\right)$. Since $x^{\prime}$ is a fixed point for the corresponding $\mathbb{C}^{*}$-action, our claim is settled.

Thus we have shown that for every $x \in X_{0}^{s s}$, the unique closed orbit in $\overline{G \cdot x}$ is contained in $X_{\vartheta}^{s s}$, whence also $G \cdot x \subset X_{\vartheta}^{s s}$ which is what we claimed.

This argumentation also yields

CoRollary 1.2.2. If, for $\vartheta \in\left(\vartheta_{i-1}, \vartheta_{i}\right)$, one has $X_{\vartheta}^{p s} \subset X_{\vartheta_{i-1}}^{p s}$, or $X_{\vartheta}^{p s} \subset X_{\vartheta_{i}}^{p s}$, then $X_{\vartheta}^{(s) s}=X_{\vartheta_{i-1}}^{(s) s}$, or $X_{\vartheta}^{(s) s}=X_{\vartheta_{i}}^{(s) s}$, respectively.

1.3. Some lemmas about Hilbert semistable curves. In the rest of this paper, we will freely make use of the fact that, if $C$ is a curve without embedded components, then the restriction map $\mathcal{E} \longrightarrow \bigoplus_{i=1}^{s} \mathcal{E}_{C_{i}}$ is injective for every locally free (or more generally depth 1 ) sheaf $\mathcal{E}$ on $C$, where the $C_{i}, i=1, \ldots, s$, are the components of $C$.

Based on ideas of the papers [5] and [11], we will now draw some consequences from the Hilbert semistability of curves. For this, fix $d, g$, and $r$ as before, and let $\mathfrak{H}_{d, g}$ be the Hilbert scheme of all closed subschemes of $\mathfrak{G}$ with Hilbert polynomial $P(m)=m d+1-g$. The notation $C \in \mathfrak{H}_{d, g}$ means that $C$ is a closed subscheme of $\mathfrak{G}$ with Hilbert polynomial $P(m)$. For any such $C$, the objects $E_{C}$ and $\psi_{C}^{m}$ are defined as in the introduction. First, since $\mathfrak{H}_{d, g}$ is projective, and $\psi_{C}^{1}\left(\bigwedge^{r} W\right) \subset H^{0}\left(\bigwedge^{r} E_{C}\right)$ is a very ample linear system, we can find an $m_{0}^{\prime}$, such that the map $\psi_{C}^{m}$ is surjective for all $m \geq m_{0}^{\prime}$ and for all $C \in \mathfrak{H}_{d, g}$. Hence, for $m \geq m_{0}^{\prime}$ and $C \in \mathfrak{H}_{d, g}$, the homomorphism $\varphi_{C}^{m}$ is also defined, and we may investigate the concept of $m$-Hilbert semistability for $C$. We set $V_{2}^{m}:=\bigwedge^{P(m)}\left(S^{m} \bigwedge^{r} W\right)$.

LEMMA 1.3.1. There is an $m_{0}^{\prime \prime} \geq m_{0}^{\prime}$, such that for every $m \geq m_{0}^{\prime \prime}$ and every $C \in \mathfrak{H}_{d, g}$ the following holds: A subspace $W_{0} \subset \operatorname{ker}\left(W \rightarrow H^{0}\left(E_{C \mid C_{\text {red }}}\right)\right)$ gives rise to a one parameter subgroup $\lambda$ of $\mathrm{SL}(W)$ with $\mu_{\mathcal{O}_{\mathbb{P}\left(V_{2}^{m}\right)}(1)}\left(\lambda,\left[\varphi_{C}^{m}\right]\right)<0$. Here, $C_{\text {red }}$ stands for the reduced subscheme of $C$.

Proof. Choose a basis $v_{1}, \ldots, v_{i_{0}}$ for $W_{0}$, complete it to a basis $v_{1}, \ldots, v_{p}$ of $W$, and define $\lambda$ w.r.t. this basis by the weight vector $\left(i_{0}-p, \ldots, i_{0}-p, i_{0}, \ldots, i_{0}\right)$ where $i_{0}-p$ occurs $i_{0}$-times. Set $W_{1}:=\left\langle v_{i_{0}+1}, \ldots, v_{p}\right\rangle$. We obtain a splitting $\bigwedge^{r} W=\bigwedge^{r} W_{1} \oplus \widetilde{\Lambda}$. The image of $\widetilde{\Lambda}$ in $H^{0}\left(\bigwedge^{r} E_{C}\right)$ lies in the kernel of the reduction $H^{0}\left(\bigwedge^{r} E_{C}\right) \longrightarrow$ $H^{0}\left(\bigwedge^{r} E_{C \mid C_{\text {red }}}\right)$, in particular, the image of $S^{m} \widetilde{\Lambda}$ in $H^{0}\left(\left(\bigwedge^{r} E_{C}\right)^{\otimes m}\right)$ is zero for all $m$ greater than some constant $\widetilde{m}$. For those $m$, the minimum weight of an eigenvector 
in $S^{m} \bigwedge^{r} W$ with non-zero image in $H^{0}\left(\left(\bigwedge^{r} E_{C}\right)^{\otimes m}\right)$ is $\widetilde{m}\left(i_{0}-p\right)+(m-\widetilde{m}) i_{0}=$ $m i_{0}-\widetilde{m} p \geq m-\widetilde{m} p$, i.e., for $m>\widetilde{m} p$ we will definitely have $\mu_{\mathcal{P}_{\mathbb{P}\left(V_{2}^{m}\right)}(1)}\left(\lambda,\left[\psi_{C}^{m}\right]\right)<0$. By the projectivity of $\mathfrak{H}_{d, g}$, we can choose $m_{0}^{\prime \prime}$ such that $m_{0}^{\prime \prime} \geq \widetilde{m}+1$ for every curve $C \in \mathfrak{H}_{d, g}$.

The rest of this section will be devoted to prove a technical key result. A curve $\widehat{C}$ will be called a tree-like curve, if it satisfies the following conditions

- $\widehat{C}$ is reduced, every irreducible component is smooth, and all intersections are ordinary double points.

- The graph $\Gamma_{\widehat{C}}$ is a tree. Here, $\Gamma_{\widehat{C}}$ is the graph with vertices $\left\{C_{0}, \ldots, C_{s}\right\}$, the irreducible components of $\widehat{C}$, and $C_{i}$ and $C_{j}$ are connected by an edge if and only if they meet.

We will call a vertex $C_{i}$ an end, if there is only one edge at $C_{i}$. We will assume from now on that all irreducible components of $\widehat{C}$ except $C_{0}$ are rational and that the genus of $\widehat{C}$ is $g$. Suppose we are given a quotient $W \otimes \mathcal{O}_{\widehat{C}} \longrightarrow E$ where $E$ is a vector bundle of rank $r$ and degree $d$ and $\operatorname{dim} W=d+r(1-g)$. We label the vertex $C_{i}$ by $d_{i}:=\operatorname{deg} E_{\mid C_{i}}, i=0, \ldots, s$, and set $d^{\prime}:=d-d_{0}$. Observe that, for $i \geq 1$, $E_{\mid C_{i}} \cong \mathcal{O}_{\mathbb{P}_{1}}\left(a_{1}\right) \oplus \cdots \oplus \mathcal{O}_{\mathbb{P}_{1}}\left(a_{r}\right)$ with $a_{1} \geq \cdots \geq a_{r} \geq 0$ and $\sum a_{j}=d_{i}$. Suppose the induced homomorphism $W \longrightarrow H^{0}(E)$ is injective. Let $H^{0}(E) \subset \bigoplus_{i=0}^{m} H^{0}\left(E_{\mid C_{i}}\right)$ be the canonical injection. Let $C_{i}, i \geq 1$, be an end (this exists), and set $W_{i}:=\operatorname{ker}(W \rightarrow$ $\bigoplus_{j \neq i} H^{0}\left(E_{\mid C_{j}}\right)$, i.e., $W_{i}=W \cap H^{0}\left(E_{\mid C_{i}}\right) \subset H^{0}\left(E_{\mid C_{i}}\left(-c_{i}\right)\right), c_{i}$ the point of intersection of $C_{i}$ with the rest of the curve. Then, $\operatorname{dim} W_{i} \leq d_{i}$, i.e., $\operatorname{dim}\left(W / W_{i}\right) \geq d-d_{i}$. By removing $C_{i}$ we obtain a new tree like curve $\widehat{C}^{\prime}$ whose graph $\Gamma_{\widehat{C}^{\prime}}$ is $\Gamma_{\widehat{C}}$ with the vertex $C_{i}$ and the edge at $C_{i}$ removed. We can therefore iterate this procedure. Set $W^{\prime}:=\operatorname{ker}\left(W \rightarrow \bigoplus_{i=1}^{m} H^{0}\left(E_{\mid C_{i}}\right)\right)$.

We will assume $\operatorname{dim}\left(W / W^{\prime}\right)=d-d^{\prime}+r(1-g)$. Then we must have had equality at each step, whence $\widetilde{W}:=\operatorname{ker}\left(W \rightarrow H^{0}\left(E_{\mid C_{0}}\right)\right)$ identifies with $H^{0}\left(E_{\widetilde{C}}\left(-p_{1}-\ldots-p_{t}\right)\right)$ where $\widetilde{C}$ is the closure of $\widehat{C} \backslash C_{0}$ in $\widehat{C}$, and $p_{1}, \ldots, p_{t}$ are the points of intersection of $C_{0}$ and $\widetilde{C}$.

Next, consider the induced morphism $f^{\prime}: \widehat{C} \longrightarrow G(W, r)$. This morphism contracts all curves $C_{i}$ with $d_{i}=0$, in particular, all ends labelled by 0 . For this reason, we can assume that no such ends are present. Under the above assumptions, the rational curves of positive degree are embedded by $f^{\prime}$, so that we can fix an end $C_{i_{0}}$ which is embedded by $f^{\prime}$. The main result we will need later is

Proposition 1.3.2. There is an $m_{0} \geq m_{0}^{\prime \prime}$, such that, for every $m \geq m_{0}$, the following conclusion is valid: In the above situation, assume there is a curve $C^{\prime} \in \mathfrak{H}_{d, g}$ such that

1. $\widehat{C}$ maps onto $C_{\mathrm{red}}^{\prime}$, the reduction of $C^{\prime}$.

2. $C^{\prime}$ is generically reduced along the image of $C_{i_{0}}$.

3. The induced map $W \rightarrow H^{0}\left(E_{C^{\prime} \mid C_{\text {red }}^{\prime}}\right)$ is injective.

Let $C_{0}^{\prime}$ be the component $f^{\prime}\left(C_{i_{0}}\right)$ of $C_{\text {red }}^{\prime}$, and $R$ the union of the remaining components of $C_{\mathrm{red}}^{\prime}$. Then we find a subspace $W_{0} \subset \operatorname{ker}\left(W \rightarrow H^{0}\left(E_{C^{\prime} \mid R}\right)\right)$, such that, for a one parameter subgroup $\lambda$ of $\mathrm{SL}(W)$ associated to this subspace as in the proof of Lemma 1.3.1, one gets $\mu_{\mathcal{O}_{\mathbb{P}}\left(V_{2}^{m}\right)(1)}\left(\lambda,\left[\varphi_{C^{\prime}}^{m}\right]\right)<0$.

Proof. There are canonical injective maps $\mathcal{O}_{C_{0}^{\prime}} \subset f_{*}^{\prime} \mathcal{O}_{C_{i_{0}}}$, and $\mathcal{O}_{R} \subset f_{*}^{\prime} \mathcal{O}_{\cup_{i \neq i} C_{i}}$. 
For this reason and because of the third assumption, the maps

$$
\begin{aligned}
W & \longrightarrow H^{0}\left(E_{C^{\prime} \mid C_{\text {red }}^{\prime}}\right) \\
& \longrightarrow H^{0}\left(E_{C^{\prime} \mid C_{0}^{\prime}}\right) \oplus H^{0}\left(E_{C^{\prime} \mid R}\right) \\
& \longrightarrow H^{0}\left(C_{i_{0}}, E_{\mid C_{i_{0}}}\right) \oplus H^{0}\left(\bigcup_{i \neq i_{0}} C_{i}, E_{\mid \cup_{i \neq i_{0}} C_{i}}\right)
\end{aligned}
$$

are injective, whence $\operatorname{ker}\left(W \rightarrow H^{0}\left(E_{C^{\prime} \mid R}\right)\right)$ naturally identifies with $H^{0}\left(E_{\mid C_{i_{0}}}\left(-c_{i_{0}}\right)\right)$. Recall that $E_{\mid C_{i_{0}}} \cong \mathcal{O}_{\mathbb{P}_{1}}\left(a_{1}\right) \oplus \cdots \oplus \mathcal{O}_{\mathbb{P}_{1}}\left(a_{r}\right)$ with $a_{1} \geq \cdots \geq a_{r} \geq 0$ and $\sum a_{j}=d_{i_{0}}>$ 0 , whence $a_{1} \geq 1$. We take $W_{0}=H^{0}\left(\mathcal{O}_{\mathbb{P}_{1}}\left(a_{1}-1\right)\right)$ under these identifications.

Let $\widetilde{C}_{0}^{\prime}$ be the scheme theoretic closure of the open subset $C^{\prime} \backslash R$ in $C^{\prime}$. Define $\tau:=\operatorname{dim} \operatorname{ker}\left(H^{0}\left(\mathcal{O}_{\widetilde{C}_{0}^{\prime}}\right) \rightarrow H^{0}\left(\mathcal{O}_{C_{0}^{\prime}}\right)\right)$. Let $\mathcal{L}$ be an invertible sheaf on $C^{\prime}$ and $\mathcal{L}^{\prime} \subset \mathcal{L}$ a subsheaf of $\mathcal{L}$ with support in $\widetilde{C}_{0}^{\prime}$, then

$$
H^{0}\left(\mathcal{L}_{\mid C_{0}^{\prime}}^{\prime}\right) \geq H^{0}\left(\mathcal{L}^{\prime}\right)-\tau \text {. }
$$

Now, we can apply the arguments used by Teixidor in [11], Proof of 2.4. Let $v_{1}, \ldots, v_{j_{0}}$ be a basis for $W_{0}$, complete it to a basis $v_{1}, \ldots, v_{p}$ of $W$, and let $\lambda$ be given w.r.t. basis by $\left(j_{0}-p, \ldots, j_{0}-p, j_{0}, \ldots, j_{0}\right)$. We also define $W_{1}:=\left\langle v_{j_{0}+1}, \ldots, v_{p}\right\rangle$. The statement $\mu_{\mathcal{O}_{\mathbb{F}}\left(V_{2}^{m}\right)(1)}\left(\lambda,\left[\varphi_{C^{\prime}}^{m}\right]\right)<0$ can be translated into the statement (cf. [5], [11])

$$
\frac{r m P(m)}{p}\left(p-a_{1}\right)<-\mu_{\mathcal{O}_{\mathbb{E}\left(V_{2}^{m}\right)}(1)}\left(\lambda^{\prime},\left[\varphi_{C^{\prime}}^{m}\right]\right)
$$

Here, $\lambda^{\prime}$ is the one parameter subgroup of $\operatorname{GL}(W)$ given w.r.t. the fixed basis by the weight vector $(0, \ldots, 0,1, \ldots, 1), 0$ appearing $j_{0}$-times. Moreover,

$$
\begin{aligned}
-\mu_{\mathcal{O}^{(}\left(V_{2}^{m}\right)(1)}\left(\lambda^{\prime},\left[\varphi_{C^{\prime}}^{m}\right]\right) & =r m P(m)-\sum_{k=0}^{r m-1} b_{k} \\
& \geq r m P(m)-\sum_{k=m(r-1)}^{r m-1} \tilde{b}_{k}-r m \tau .
\end{aligned}
$$

Here, $b_{k}$ and $\tilde{b}_{k}$ are the dimensions of the subspaces of $H^{0}\left(\left(\bigwedge^{r} E_{C^{\prime}}\right)^{\otimes m}\right)$ and $H^{0}\left(\left(\bigwedge^{r} E_{C^{\prime} \mid C_{0}^{\prime}}\right)^{\otimes m}\right)$ generated by the eigenspace of weight $k$ in $S^{m}\left(\bigwedge^{r} W\right)$. Note that only the space $S^{m}\left(W_{0} \otimes \bigwedge^{r-1} W_{1} \oplus \bigwedge^{r} W_{1}\right)$ yields non-zero sections in $H^{0}\left(\left(\bigwedge^{r} E_{C^{\prime} \mid C_{0}^{\prime}}\right)^{\otimes m}\right)$, so that the asserted inequality follows from (1.4). Next, by definition, for $m(r-1) \leq k<m r$, the image of the eigenspace of weight $k$ lies in $H^{0}\left(\mathbb{P}_{1}, \mathcal{O}_{\mathbb{P}_{1}}\left(m d_{i_{0}}-(m n-k)\right)\right)$, i.e., $\widetilde{b}_{k} \leq m d_{i_{0}}+k-m n+1$. The left hand side of $(1.5)$ is $m^{2} \cdot r d\left(1-a_{1} / p\right)+l_{1}(m), l_{1}(m)$ a linear polynomial, and the right hand side is bounded from below by $m^{2}\left(r d-d_{i_{0}}+1 / 2\right)+l_{2}(m)-r m \tau, l_{2}(m)$ also a linear polynomial. Negating (1.5) for large $m$ yields

$$
a_{1} \leq \frac{p}{r d}\left(d_{i_{0}}-\frac{1}{2}\right)<\frac{d_{i_{0}}}{r},
$$

a contradiction.

Now, the polynomials $l_{1}(m)$ and $l_{2}(m)$ depend only on $d, g, r$ and $d_{i_{0}}$ which leaves only finitely many possibilities after fixing $d, g$, and $r$, because $0<d_{i_{0}} \leq d$. Moreover, $\tau$ is bounded by $h^{0}\left(\mathcal{O}_{C^{\prime}}\right)-1$, so it can take only finitely many values for $C^{\prime}$ varying in $\mathfrak{H}_{d, g}$. This means that we can indeed find $m_{0}$ as asserted. 
2. Proof of the Theorem. Choose $d>d_{1}$ according to Proposition 1.1.3, fix a complex vector space $W$ of dimension $d+r(1-g)$, and let $C \hookrightarrow \mathfrak{G}=G(W, r)$ be a smooth curve of genus $g$. This provides us, on $C$, with a quotient $W \otimes \mathcal{O}_{C} \longrightarrow E_{C}$. Write $L=L_{C}$ for the line bundle $\operatorname{det} E_{C}$. Let $\mathfrak{Q}_{0}$ be the quasi projective quot scheme parametrizing all quotients $q: W \otimes \mathcal{O}_{C} \longrightarrow E$, such that

- $E$ is a vector bundle on $C$ of rank $r$ with determinant $L$

- $H^{0}(q)$ is an isomorphism

- $\bigwedge^{r} W \longrightarrow H^{0}(L)$ is surjective.

2.1. Review of Gieseker's construction of the moduli space of stable bundles. On $\mathfrak{Q}_{0} \times C$, there is the universal quotient $W \otimes \mathcal{O}_{\mathfrak{Q}_{0} \times C} \longrightarrow \mathfrak{E}_{\mathfrak{Q}_{0}}$ which provides us with $\bigwedge^{r} W \otimes \mathcal{O}_{\mathfrak{Q}_{0} \times C} \longrightarrow \bigwedge^{r} \mathfrak{E}_{\mathfrak{Q}_{0}}$. Note that $\bigwedge^{r} \mathfrak{E}_{\mathfrak{Q}_{0}} \cong \pi_{C}^{*} L \otimes \pi_{\mathfrak{Q}_{0}}^{*} \mathcal{A}$ for some $\mathrm{SL}(W)$-linearized line bundle $\mathcal{A}$ on $\mathfrak{Q}_{0}$, so that projecting the latter homomorphism to $\mathfrak{Q}_{0}$ yields

$$
\bigwedge^{r} W \otimes \mathcal{O}_{\mathfrak{Q}_{0}} \longrightarrow H^{0}(L) \otimes \mathcal{A}
$$

This homomorphism induces an injective and SL $(W)$-equivariant morphism $\iota: \mathfrak{Q}_{0} \longrightarrow$ $\mathbb{P}\left(V_{1}\right)$ with $V_{1}:=\operatorname{Hom}\left(\bigwedge^{r} W, H^{0}(L)\right)^{\vee}$. Using Corollary 1.1.2 and Proposition 1.1.3, it follows that the preimage under $\iota$ of the (semi/poly)stable points is exactly the set of quotients $q: W \otimes \mathcal{O}_{C} \longrightarrow E$ for which $E$ is a (semi/poly)stable vector bundle. Write $\mathfrak{Q}^{(s / p) s}$ for the respective sets. The induced map $\iota: \mathfrak{Q}^{s s} \longrightarrow \mathbb{P}\left(V_{1}\right)^{s s}$ is proper, from which one infers that $\mathcal{M}_{L / r}:=\mathfrak{Q}^{s s} / / \mathrm{SL}(W)$ exists.

2.2. Proof of the theorem. Set $V_{2}^{m}:=\bigwedge^{P(m)}\left(S^{m} \bigwedge^{r} W\right)$, so that, for every $m \geq 1$, we have a natural morphism

$$
j_{m}: \mathfrak{Q}_{0} \longrightarrow \mathbb{P}\left(V_{1}\right) \times \mathbb{P}\left(V_{2}^{m}\right) .
$$

REMARK 2.2.1. We remark in passing that the pullback of $\mathcal{O}(1)$ under the morphism $\mathfrak{Q}_{0} \longrightarrow \mathbb{P}\left(V_{2}^{m}\right)$ is just $\mathcal{A}^{\otimes m P(m)}$, i.e., the morphisms from $\mathfrak{Q}_{0}$ to $\mathbb{P}\left(V_{1}\right)$ and $\mathbb{P}\left(V_{2}^{m}\right)$ both give rise to the same $\mathrm{SL}(W)$-linearized line bundle on $\mathfrak{Q}_{0}$.

Let $X^{m}$ be the closure of $j_{m}\left(\mathfrak{Q}_{0}\right)$. We will now use the notation of Section 1.2. Note that for every point $x=\left(\left[x_{1}\right],\left[x_{2}\right]\right)=j_{m}\left(\left[q: W \otimes \mathcal{O}_{C} \longrightarrow E\right]\right)$ and every one parameter subgroup $\lambda$ of $\mathrm{SL}(W)$, we have

$$
m P(m) \cdot \mu_{\mathcal{O}_{\mathbb{E}\left(V_{1}\right)}(1)}\left(\lambda,\left[x_{1}\right]\right) \geq \mu_{\mathcal{O}_{\left(V_{2}^{m}\right)}(1)}\left(\lambda,\left[x_{2}\right]\right)
$$

so that in view of Prop. 1.1.3, one immediately infers

CoRollary 2.2.2. If the curve $C$ is $m$-Hilbert (semi)stable, then the vector bundle $E_{C}$ is (semi)stable.

REMARK 2.2.3. Note that this conclusion holds for every $m \geq 1$. following

By Lemma 1.2.1, $j_{m}\left(\mathfrak{Q}^{s s}\right)=\left(X^{m}\right)_{\vartheta}^{s s} \subset\left(X^{m}\right)_{\vartheta_{1}}^{s s}$. Suppose now we could prove the 
Proposition 2.2.4. Let $m_{0}$ be as in Prop. 1.3.2. Then for all $m \geq m_{0}$, the following holds true: Let $\left[q: W \otimes \mathcal{O}_{C} \longrightarrow E\right] \in \mathfrak{Q}^{s s}$, such that $E$ is a polystable vector bundle. Suppose $j_{m}([q]) \in\left(X^{m}\right)_{\vartheta}^{s s}$ for some $\vartheta \in(0, \infty)$. Then, also

$$
j_{m}([q]) \in\left(X^{m}\right)_{\vartheta}^{p s} .
$$

In this case, by Corollary 1.2.2, $j_{m}\left(\mathfrak{Q}^{(s) s}\right)=\left(X^{m}\right)_{\vartheta_{1}}^{(s) s}$. Using (2.1) and Lemma 1.2.1 again, we also get $\left(X^{m}\right)_{\vartheta}^{(s) s}=\left(X^{m}\right)_{\vartheta_{1}}^{(s) s}$ for all $\vartheta \in\left(\vartheta_{1}, \vartheta_{2}\right)$. Now, iterating this argumentation, yields the conclusion

$$
\left(X^{m}\right)_{\infty}^{(s) s}=j_{m}\left(\mathfrak{Q}^{(s) s}\right)
$$

which is just a reformulation of the assertion of the theorem.

2.3. Proof of Proposition 2.2.4. Let $\lambda$ be a one parameter subgroup of $\mathrm{SL}(W)$, such that $x_{0}:=\lim _{z \rightarrow 0} j_{m}([q]) \cdot \lambda(z)$ exists in $\left(X^{m}\right)_{\vartheta}^{s s}$, but such that $j_{m}([q])$ is not a fixed point for the corresponding $\mathbb{C}^{*}$-action. We must describe $x_{0}=\left(\left[x_{1}\right],\left[x_{2}\right]\right)$ more explicitly to derive a contradiction. First, by assumption, we have a morphism $\mathbb{C}^{*} \longrightarrow \mathfrak{Q}^{s s}$. This corresponds to a family $W \otimes \mathcal{O}_{\mathbb{C}^{*} \times C} \longrightarrow \mathcal{E}_{\mathbb{C}^{*}}$. This family can be extended to a family of quotients $W \otimes \mathcal{O}_{\mathbb{C} \times C} \longrightarrow \mathcal{E}_{\mathbb{C}}$ where $\mathcal{E}_{\mathbb{C}}$ is a $\mathbb{C}$-flat family of coherent sheaves of rank $r$ with determinant $L$ on $C$. Note that the flatness over $\mathbb{C}$ implies that $\mathcal{E}_{\mathbb{C}}$ is torsion free as $\mathcal{O}_{\mathbb{C} \times C}$-module. Set $E_{\mathbb{C}}:=\mathcal{E}_{\mathbb{C}}^{\vee \vee}$. This is a reflexive sheaf on the smooth surface $\mathbb{C}^{*} \times C$, whence it is locally free and thus flat over $\mathbb{C}$. This gives a family

$$
W \otimes \mathcal{O}_{\mathbb{C} \times C} \longrightarrow E_{\mathbb{C}}
$$

REMARK 2.3.1. Let us remind the reader of some features of this construction.

1. The kernel of the homomorphism $\mathcal{E}_{\mathbb{C} \mid\{0\} \times C} \longrightarrow E_{\mathbb{C} \mid\{0\} \times C}$ is exactly the torsion $\mathcal{T}$ of $\mathcal{E}_{\mathbb{C} \mid\{0\} \times C}$.

2. Since $W \otimes \mathcal{O}_{C}$ generically generates $E_{\mathbb{C} \mid\{0\} \times C}$, we see $\operatorname{dim}_{\mathbb{C}}(\mathcal{T}) \leq \operatorname{dim} W-r=$ $d-r g<d$, thus $\operatorname{deg}\left(\mathcal{E}_{\mathbb{C} \mid\{0\} \times C} / \mathcal{T}\right)>0$ has positive degree, and since there is a surjection $W \otimes \mathcal{O}_{C} \longrightarrow \mathcal{E}_{\mathbb{C} \mid\{0\} \times C} / \mathcal{T}$, the rational map $C \rightarrow \mathfrak{G}$ induced by $W \otimes C \longrightarrow E_{\mathbb{C} \mid\{0\} \times C}$ is not constant.

3. Set $\widetilde{W}:=W / \operatorname{ker}\left(W \rightarrow H^{0}\left(\mathcal{E}_{\mathbb{C} \mid\{0\} \times C}\right)\right)$. Then, $\operatorname{dim} \operatorname{Im}\left(W \rightarrow H^{0}\left(E_{\mathbb{C} \mid\{0\} \times C}\right)\right)$ $=\operatorname{dim}(\widetilde{W})-d^{\prime}$, by 1 .

From this discussion, we deduce that the homomorphism

$$
\bigwedge^{r} W \otimes \mathcal{O}_{\mathbb{C} \times C} \rightarrow \bigwedge^{r} E_{\mathbb{C}}
$$

is surjective outside a finite set of points $p_{1}, \ldots, p_{t}$ located on $\{0\} \times C$ where $t \leq d^{\prime}$. In particular, there is a rational map

$$
h: \mathbb{C} \times C \rightarrow \mathfrak{G}
$$

defined outside $\left\{p_{1}, \ldots, p_{t}\right\}$. By blowing up the points $p_{1}, \ldots, p_{t}$ and possibly some infinitely near ones (see [1], II.7), we arrive at a smooth surface $\widehat{S}$ together with a morphism

$$
\widehat{h}: \widehat{S} \longrightarrow \mathfrak{G} .
$$


REMARK 2.3.2. The map $\bigwedge^{r} W \otimes \mathcal{O}_{\{0\} \times C} \longrightarrow H^{0}(L)=H^{0}\left(\operatorname{det} E_{\mathbb{C} \mid\{0\} \times C}\right)$ defines the point $\left[x_{1}\right] \in \mathbb{P}\left(V_{1}\right)$. As in the proof of 1.3 .1 , every subspace $W_{0} \subset \operatorname{ker}(W \rightarrow$ $\left.H^{0}\left(E_{\mathbb{C} \mid\{0\} \times C}\right)\right)$ yields a one parameter subgroup $\lambda$ of $\mathrm{SL}(W)$ with $\mu_{\mathcal{O}_{\mathbb{P}\left(V_{1}\right)}(1)}\left(\lambda,\left[x_{1}\right]\right)<$ 0 .

The composite morphism $\widehat{S} \longrightarrow \mathbb{C}$ is still flat, and an easy inductive argument shows that the fibre $\widehat{C}$ over $\{0\}$ is a tree-like curve with $C$ as its only non-rational component.

Next, observe that by Butler's results 1.1.2, the morphism $\mathbb{C}^{*} \times C \longrightarrow \mathbb{C}^{*} \times$ $\mathfrak{G} \hookrightarrow \mathbb{C}^{*} \times \mathbb{P}\left(\bigwedge^{r} W\right)$ is an embedding and consequently corresponds to a morphism $\mathbb{C}^{*} \longrightarrow \mathfrak{H}_{d, g}$. By extending this morphism to a morphism $\mathbb{C} \longrightarrow \mathfrak{H}_{d, g}$, we get another surface $S^{\prime}$ equipped with a flat morphism to $\mathbb{C}$. Observe that the flatness over $\mathbb{C}$ together with the fact that $S^{\prime} \times \mathbb{C} \mathbb{C}^{*}$ is integral implies that $S^{\prime}$ is also integral ([6], III, Prop.9.7). Moreover, by our construction, there a morphsim $f: \widehat{S} \longrightarrow S^{\prime}$ which factorizes over $\widehat{S} \longrightarrow \widetilde{S}^{\prime}, \widetilde{S}^{\prime}$ the normalization of $S^{\prime}$. The latter morphism just being the contraction of some rational curves with negative self-intersection, the morphism $f: \widehat{S} \longrightarrow S^{\prime}$ is proper.

Now, write $\mathbb{C}=\operatorname{Spec} \mathbb{C}[T]$, and denote by $T$ also the induced element in the function field $K(\widehat{S})=K\left(S^{\prime}\right)$. Let $C^{\prime}$ be the fibre of $S^{\prime}$ over $\{0\}$. We will have to compare $\widehat{C}$ and $C^{\prime}$. For this let $[\widehat{C}]$ and $\left[C^{\prime}\right]$ be the Weil divisor classes of those curves. By definition $\widehat{C}$ and $C^{\prime}$ are the Cartier $\operatorname{divisors} \operatorname{div}(T)$, taken on $\widehat{S}$ and $S^{\prime}$, respectively. Proposition 1.4 in [3] thus shows that $f_{*}[\widehat{C}]=\left[C^{\prime}\right]$ on the cycle level. The upshot of this discussion is that, if we can show that every rational curve in $\widehat{C}$ which is not contracted is mapped injectively to $\mathfrak{G}$, the only component of $C^{\prime}$ which is possibly not generically reduced is $f(C)$, the ultimate goal being to apply Proposition 1.3.2.

Anyway, at this stage we know that the curve $C^{\prime} \in \mathfrak{H}_{d, g}$ supplies $\left[x_{2}\right]$ in $\mathbb{P}\left(V_{2}^{m}\right)$. Therefore, we can look at some destabilizing one parameter subgroups.

LEMma 2.3.3. For $m \geq m_{0}$, the homomorphism $W \longrightarrow H^{0}\left(E_{C_{\mathrm{red}}^{\prime}}\right)$ must be injective.

Proof. Observe that a subspace $W_{0}$ of $\operatorname{ker}\left(W \rightarrow H^{0}\left(E_{C_{\text {red }}^{\prime}}\right)\right)$ gives by Lemma 1.3.1 and Remark 2.3.2 rise to a one parameter subgroup $\lambda$ with both $\mu_{\mathcal{O}_{\mathbb{F}\left(V_{1}\right)}(1)}\left(\lambda,\left[x_{1}\right]\right)<0$ and $\mu_{\mathcal{O}_{\mathbb{F}\left(V_{2}^{m}\right)(1)}}\left(\lambda,\left[x_{2}\right]\right)<0$, in contradiction to the semistability of $x_{0}$.

The induced morphism $f^{\prime}: \widehat{C} \longrightarrow C_{\text {red }}^{\prime}$ is surjective, so that there are injections $\mathcal{O}_{C_{\text {red }}^{\prime}} \subset f_{*}^{\prime} \mathcal{O}_{\widehat{C}}$, and $E_{C^{\prime} \mid C_{\text {red }}^{\prime}} \subset E_{C^{\prime} \mid C_{\text {red }}^{\prime}} \otimes f_{*}^{\prime} \mathcal{O}_{\widehat{C}}=f_{*}^{\prime} f^{\prime *} E_{C^{\prime} \mid C_{\text {red }}^{\prime}}$. The composite $W \stackrel{\text { red }}{\longrightarrow} H^{0}\left(E_{C^{\prime} \mid C_{\text {red }}^{\prime}}\right) \subset H^{0}\left(f^{\prime *} E_{C^{\prime} \mid C_{\text {red }}^{\prime}}\right)$ is thus injective by Lemma 2.3.3. Therefore, in Remark 2.3.1, 3., the space $\widetilde{W}$ equals $W / H^{0}(\mathcal{T})$ and, thus, has dimension $d-$ $d^{\prime}+r(1-g)$. Now, one immediately checks that we are exactly in the position to apply Proposition 1.3.2. Since the subspace $W_{0}$ used to destabilize $\left[x_{2}\right]$ lies in the kernel of $W \rightarrow H^{0}\left(E_{\mathbb{C} \mid\{0\} \times C}\right)$, we find again a one parameter subgroup $\lambda$ of $\operatorname{SL}(W)$ with $\mu_{\mathcal{O}_{\mathbb{E}\left(V_{1}\right)}(1)}\left(\lambda,\left[x_{1}\right]\right)<0$ and $\mu_{\mathcal{O}_{\mathbb{E}\left(V_{2}^{m}\right)}(1)}\left(\lambda,\left[x_{2}\right]\right)<0$, contradicting the assumptions made on $x_{0}$. 


\section{REFERENCES}

[1] A. Beauville, Surfaces Algébriques Complexes, Astérisque 54, 1978.

[2] D. Butler, Normal generation of vector bundles over a curve, J. Diff. Geom., 39 (1994), pp. $1-34$.

[3] W. FulToN, Intersection Theory (Second Ed.), Springer, 1998.

[4] D. GiEseKer, A degeneration of the moduli space of stable bundles, J. Diff. Geom., 19 (1984), pp. 1-29.

[5] D. GIESEKER AND I. MORRISON, Hilbert stability of rank-two bundles on curves, J. Diff. Geom., 19 (1984), pp. 173-206.

[6] R. Hartshorne, Algebraic Geometry, Springer, 1977.

[7] D. Huybrechts and M. Lehn, The Geometry of the Moduli Spaces of Sheaves, Vieweg, 1997.

[8] J. LEPotIER, Lectures on Vector Bundles, CUP, 1997.

[9] R. Pandharipande, A compactification over $\bar{M}_{g}$ of the universal moduli space of slopesemistable vector bundles, J. AMS, 9 (1996), pp. 425-71.

[10] N. RESSAYRE, The GIT-equivalence for G-bundles, Geom. Dedicata, 81 (2000), pp. 295-324.

[11] M. TEIXIDOR, Compactifications of moduli spaces of (semi)stable bundles on singular curves: two points of view, in Serrano memorial volume, Collect. Math., 49 (1998), pp. 527-48.

[12] M. TeIXIDor, Curves in Grassmannians, Proc. Amer. Math. Soc., 126 (1998), pp. 1597-1603.

[13] M. Thaddeus, Geometric Invariant Theory and flips, J. AMS, 9 (1996), pp. 691-723. 